2. H. Cartan, Séminaire de Topologie de l'E.N.S., Paris, 1954-1955 (Notes polycopiées).

3. E. Halpern, On the structure of hyperalgebras. Class 1 Hopf algebras, Portugal. Math. vol. 17 fasc. 6 (1959).

4. J. Leray, Sur la forme des espaces topologiques et sur les points fixes des representations, J. Math. Pures Appl. vol. 54 (1945).

5. J. C. Moore, Mimeographed notes of the Algebraic Topology Seminar, Princeton, 1957.

6. H. Samelson, Beiträge zur Topologie der Gruppen-Mannigfaltigkeiten, Ann. of Math. vol. 42 (1941).

7. N. E. Steenrod, Cyclic reduced powers of cohomology classes, Proc. Nat. Acad. Sci. U.S.A. vol. 39 (1953).

UNIVERSITY OF Michigan

\title{
SOME GLOBAL PROPERTIES OF HYPERSURFACES ${ }^{1}$
}

ROBERT E. STONG

1. Introduction. The translation theorem of Hopf [1] has been extended by Hsiung [2] and Voss [4] independently to hypersurfaces and by Hsü [3] to other elementary transformations. The purpose of this paper is to extend to hypersurfaces in $(n+1)$-dimensional Euclidean space some results obtained by Hsü [3] for the case $n=2$.

All hypersurfaces mentioned will be assumed to be twice differentiably imbedded in an $(n+1)$-dimensional Euclidean space $E^{n+1}(n+1 \geqq 3)$. The notation used will be that of Hsiung [2]. In particular, $X, N, M_{1}, A$ denote the position vector, unit inner normal, first mean curvature, and area for the hypersurface $V^{n}$. Corresponding quantities for other hypersurfaces will be denoted by ${ }^{*}$, or by primes.

Considerable use will be made of the vector product defined by Hsiung [2]. Namely, if $i_{1}, \cdots, i_{n+1}$ denotes a fixed frame of mutually orthogonal unit vectors and $A_{1}, \cdots, A_{n}$ are $n$ vectors whose components in this frame are $A_{i}^{\alpha}(i=1, \cdots, n ; \alpha=1, \cdots, n+1)$, the vector product is defined by

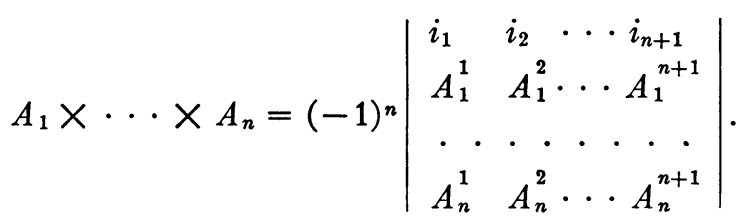

Presented to the Society, June 20, 1959; received by the editors April 20, 1959.

1 This is a portion of a master's thesis at the University of Oklahoma directed by Professor T. K. Pan. 
Further, in any vector product involving differentials, the exterior convention for multiplication of differentials will be observed. As in [2], the following formulae will be used:

$$
\begin{aligned}
d X \times \cdots \times d X & =n ! N d A, \\
d X \times \cdots \times d X \times d N & =-n ! M_{1} N d A .
\end{aligned}
$$

A closed nonselfintersecting hypersurface $V^{n}$ is said to be convex with respect to the point $O$ if: (1) every straight line through $O$ meets $V^{n}$ in at most two points, and (2) the mapping $f: V^{n} \rightarrow V^{n}$ which takes a point of $V^{n}$ into the other point on the same line through $O$ is a differentiable homeomorphism.

The following results will be obtained:

Theorem 1. Let $V^{n}, V^{*_{n}}$ be two closed orientable hypersurfaces. Suppose there is a differentiable homeomorphism $f: V^{n} \rightarrow V^{*_{n}}$ such that: (1) each straight line $P P^{*}$ joining corresponding points $P$ and $P^{*}$ passes through a fixed point $O ;(2)$ with $O$ as origin, the position vectors and first mean curvatures are related to each other by either (a) $M_{1}^{*} X^{*}=M_{1} X$ or by (b) $M_{1}^{*} X^{*}=-M_{1} X$ throughout $V^{n}$ and $V^{*_{n}}$; and (3) $V^{n}$ and $V^{*_{n}}$ contain no pieces of hypercones with vertex $O$. Then $f$ is a homothetic transformation with center $O$ and positive or negative constant of proportionality as (a) or (b) holds.

THEOREM 2. Let $V^{n}$ and $V^{*_{n}}$ be two closed orientable hypersurfaces. Suppose there is a differentiable homeomorphism $f: V^{n} \rightarrow V^{* n}$ such that: (1) there is a fixed point $O$ such that for every pair of points $P, Q$ of $V^{n}$ and their images $P^{*}, Q^{*}$ in $V^{* n}$, the angles $P O Q$ and $P^{*} O Q^{*}$ are equal; (2) with $O$ as origin $M_{1}^{*} X^{*}$ and $M_{1} X$ are equal in magnitude; and (3) neither $V^{n}$ nor $V^{* n}$ contains pieces of hypercones with vertex $O$. Then $f$ is a similarity transformation with $O$ as center of similitude.

Theorem 3. Let $V^{n}, V^{* n}$ be two closed orientable hypersurfaces. Suppose there is a differentiable homeomorphism $f: V^{n} \rightarrow V^{*_{n}}$ such that: (1) each straight line $P P^{*}$ joining corresponding points $P$ and $P^{*}$ passes through a fixed point $O ;(2)$ with $O$ as origin, the position vectors, normals, and first mean curvatures are related by either

$$
M_{1}^{*} X^{*}=-\left(M_{1}+2 \frac{X \cdot N}{X \cdot X}\right) X
$$

or by

$$
M_{1}^{*} X^{*}=\left(M_{1}+2 \frac{X \cdot N}{X \cdot X}\right) X
$$


throughout $V^{n}$ and $V^{*_{n}}$; and (3) neither $V^{n}$ nor $V^{*_{n}}$ contains the point $O$ or pieces of hypercones with vertex $O$. Then $f$ is an inversion with center $O$ and real or imaginary radius of inversion as (a) or (b) respectively holds.

2. Integral formulae. Let $V^{n}$ be an orientable hypersurface with closed boundary $V^{n-1}$. Further suppose $V^{n}$ does not contain $O$. Let $k$ be a differentiable function on $V^{n}$ which is finite and nonzero. Let a hypersurface $V^{*_{n}}$ be defined by

$$
X^{*}=k X,
$$

with

$$
k M_{1}^{*}=M_{1} .
$$

Applying (1.1) and noting that any vector product having two or more factors of $X$ vanishes, one finds

$$
n ! N^{*} d A^{*}=n ! k^{n} N d A+n k^{n-1}(X d k \times d X \times \cdots \times d X) .
$$

Taking the scalar product of (2.3) with $M_{1}^{*} X^{*}=M_{1} X$ and using $X \cdot(X d k \times d X \times \cdots \times d X)=0$ yield

$$
M_{1}^{*} X^{*} \cdot N^{*} d A^{*}=k^{n} M_{1} X \cdot N d A .
$$

Let $\alpha=N \cdot(X \times d X \times \cdots \times d X)$ and $\beta=N^{*} \cdot(X \times d X \times \cdots \times d X)$. Then it follows that

$$
d \alpha=n ! M_{1} X \cdot N d A+n ! d A .
$$

From (1.2) one has

$$
\begin{aligned}
-n ! M_{1}^{*} X^{*} \cdot N^{*} d A^{*} & =X^{*} \cdot\left(d N^{*} \times d X^{*} \times \cdots \times d X^{*}\right) \\
& =k^{n} X \cdot\left(d N^{*} \times d X \times \cdots \times d X\right),
\end{aligned}
$$

so that

$$
\begin{aligned}
d \beta & =n ! k^{-n} M_{1}^{*} X^{*} \cdot N^{*} d A^{*}+n ! N^{*} \cdot N d A \\
& =n ! M_{1} X \cdot N d A+n ! N^{*} \cdot N d A .
\end{aligned}
$$

Subtracting (2.5) from (2.4) gives

$$
n !\left(1-N^{*} \cdot N\right) d A=d(\alpha-\beta) .
$$

Integrating (2.6) and applying Stokes' formula yields

$$
\begin{aligned}
n ! \int_{V^{n}}\left(1-N^{*} \cdot N\right) d A & \\
= & \int_{V^{n-1}}\left(N-N^{*}\right) \cdot(X \times d X \times \cdots \times d X) .
\end{aligned}
$$


If $(2.1)$ is replaced by

$$
X^{*}=-k X,
$$

with (2.2) unchanged, one finds as above that

$$
\begin{aligned}
n ! \int_{V^{n}}\left(1+N^{*} \cdot N\right) & d A \\
& =\int_{V^{n-1}}\left(N+N^{*}\right) \cdot(X \times d X \times \cdots \times d X) .
\end{aligned}
$$

\section{Proofs of theorems.}

Proof of Theorem 1. Let $M_{1}^{*} X^{*}=M_{1} X$ and let $X^{*}=k X$ where $k=M_{1} / M_{1}^{*}$.

CAse I. $O \notin V^{n}$ and $O \notin V^{* n}$. Then $k \neq 0, \infty$. Since $V^{n}$ is closed, $V^{n-1}$ is empty. Formula (2.7) then applies, giving

$$
\int_{V^{n}}\left(1-N^{*} \cdot N\right) d A=0 .
$$

$d A$ is of fixed sign and $1-N^{*} \cdot N=1-\cos \beta \geqq 0$, where $\beta$ denotes the angle between $N$ and $N^{*}$. (3.1) then implies $1-N^{*} \cdot N=0$ or $N^{*}=N$.

Inasmuch as $N^{*} \cdot d X^{*}=N \cdot d X=0$, one has

$$
\begin{aligned}
(N \cdot X) d k & =N \cdot\left(d X^{*}-k d X\right) \\
& =N^{*} \cdot d X^{*}-k N \cdot d X \\
& =0 .
\end{aligned}
$$

The set $S$ of points of $V^{n}$ for which $X \cdot N=0$ can have no interior points, since $S$ would then contain a piece of a hypercone with vertex $O$. Thus $V^{n}-S$ is dense in $V^{n}$. Hence $d k$ is a continuous function on $V^{n}$ which vanishes on a dense subset, so $d k=0$ everywhere. Then $k$ is constant and since $N^{*}=N, k$ must be positive.

CASE II. $O \in V^{n}$ or $O \in V^{* n}$. One may assume $O \in V^{n}$ without loss of generality. Let $U$ be any open set of $V^{n}$ containing $O$, and let $V$ be a neighborhood of $O$ which is contained in $U$. Let $V^{\prime}$ be the boundary of $V$ (and $V^{n}-V$ ). Since $\left(1-N^{*} \cdot N\right) d A$ does not change sign,

$$
\left|\int_{V^{n}-U}\left(1-N^{*} \cdot N\right) d A\right| \leqq\left|\int_{V^{n}-V}\left(1-N^{*} \cdot N\right) d A\right|,
$$

and by (2.7)

$$
\left|\int_{V^{n}-V}\left(1-N^{*} \cdot N\right) d A\right|=\frac{1}{n !}\left|\int_{V^{\prime}}\left(N-N^{*}\right) \cdot(X \times d X \times \cdots \times d X)\right|,
$$


which can be made small by making $V$ small. The right member of (3.2) is fixed, however, so that

$$
\int_{V^{n} \_U}\left(1-N^{*} \cdot N\right) d A=0 .
$$

As in Case $\mathrm{I}, k$ is then a positive constant in $V^{n}-U$ for any $U$. By continuity, $k$ is then a positive constant throughout $V^{n}$.

If instead, $M_{1}^{*} X^{*}=-M_{1} X$, let $X^{*}=-k X$ with $k=M_{1} / M_{1}^{*}$. From $\left(2.7^{\prime}\right)$ one has

$$
\int_{V^{n}}\left(1+N^{*} \cdot N\right) d A=0
$$

so

$$
N^{*}=-N
$$

Proceeding as before, $k$ must be a positive constant.

REMARK. Consideration of (2.7) and $\left(2.7^{\prime}\right)$ gives immediately that one may replace the condition that $V^{n}$ and $V^{*_{n}}$ be closed by: $V^{n}, V^{*_{n}}$ have closed boundaries $V^{n-1}$ and $V^{* n-1}$ such that at corresponding points of the boundaries (a) $N^{*}=N$ or (b) $N^{*}=-N$ respectively, and Theorem 1 still holds.

CoROllary. Let $V^{n}$ be a closed orientable hypersurface which is convex with respect to a fixed point $O$. If with $O$ as origin $M_{1}^{\prime} X^{\prime}=-M_{1} X$, where $X^{\prime}$ denotes the image of $X$ under $f: V^{n} \rightarrow V^{n}$, then $V^{n}$ is symmetric with respect to $O$.

By Theorem 1, $f$ is a homothetic transformation with negative constant of proportionality $-k$ and center $O$. Then $f \circ f=$ identity, so $k^{2}=1$ or $k=1$.

Proof of Theorem 2. Let $g(X)=(|X| f(X) /|f(X)|)$. Since $g$ preserves angles and magnitudes, $g$ is given by a motion (possibly improper) which leaves $O$ fixed. Then $f g^{-1}: V^{\prime n}=g\left(V^{n}\right) \rightarrow V^{*_{n}}$ satisfies the conditions of Theorem 1. $f=\left(f g^{-1}\right) g$ is then a motion followed by a homothetic transformation with center $O$. Thus $f$ is a similarity with center $O$.

Proof of Theorem 3. Let $g: E^{n+1}-\{O\} \rightarrow E^{n+1}-\{O\}$ denote the inversion with respect to the unit hypersphere. Let $V^{\prime n}=g\left(V^{n}\right)$. By straightforward calculation, one finds

$$
M_{1}^{\prime} X^{\prime}=-\left(M_{1}+2 \frac{X \cdot N}{X \cdot X}\right) X
$$


Thus one has (a) $M_{1}^{\prime} X^{\prime}=M_{1}^{*} X^{*}$ or (b) $M_{1}^{\prime} X^{\prime}=-M_{1}^{*} X^{*}$ respectively. Applying Theorem $1, f^{-1}$ is a homothetic transformation with center $O . f=\left(f g^{-1}\right) g$ is then an inversion with the given properties.

REMARK. If $V^{n}, V^{*_{n}}$ have closed boundaries $V^{n-1}$ and $V^{*_{n-1}}$ and if at corresponding points of the boundaries one has, respectively, (a) $N^{*}=-N+2(X \cdot N / X \cdot X) X$ or (b) $N^{*}=N-2(X \cdot N / X \cdot X) X$, the theorem also holds.

Since one has (a) $N^{*}=N^{\prime}$ or (b) $N^{*}=-N^{\prime}$ respectively, the remark of Theorem 1 gives the desired result.

COROLlARY. If $V^{n}$ is a closed orientable hypersurface which is convex with respect to a point $O$ not in $V^{n}$ and if with $O$ as origin,

$$
M_{1}=-X \cdot N / X \cdot X,
$$

then $V^{n}$ is a hypersphere.

Since $M_{1} X=-\left(M_{1}+2 X \cdot N / X \cdot X\right) X$, each point of $V^{n}$ is invariant under the inversion in a hypersphere of real radius with center $O$. Thus $V^{n}$ is this hypersphere.

\section{REFERENCES}

1. H. Hopf and K. Voss, Ein Satz aus der Flächentheorie im Grossen, Arch. Math. vol. 3 (1952) pp. 187-192.

2. C. C. Hsiung, Some global theorems on hypersurfaces, Canad. J. Math. vol. 9 (1957) pp. 5-14.

3. C. S. Hsü, Characterization of some elementary transformations, Proc. Amer. Math. Soc. vol. 10 (1959) pp. 324-328.

4. K. Voss, Einige differentialgeometrische Kongruenzsätze für geschlossene Flächen und Hyperflächen, Math. Ann. vol. 131 (1956) pp. 180-218.

UNIVERSITY OF OKLAHOMA 